\title{
The novel BH-3 mimetic apogossypolone induces Beclin-1- and ROS-mediated autophagy in human hepatocellular carcinoma cells
}

\author{
This article has been corrected since Online Publication and a corrigendum has also been published
}

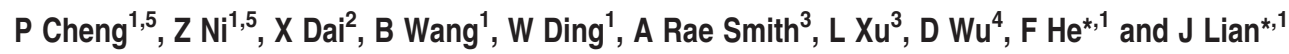

Apogossypolone (ApoG2), a novel derivative of gossypol, exhibits superior antitumor activity in Bcl-2 transgenic mice, and induces autophagy in several cancer cells. However, the detailed mechanisms are not well known. In the present study, we showed that ApOG2 induced autophagy through Beclin-1- and reactive oxygen species (ROS)-dependent manners in human hepatocellular carcinoma (HCC) cells. Incubating the HCC cell with ApoG2 abrogated the interaction of Beclin-1 and Bcl-2/xL, stimulated ROS generation, increased phosphorylation of ERK and JNK, and HMGB1 translocation from the nucleus to cytoplasm while suppressing mTOR. Moreover, inhibition of the ROS-mediated autophagy by antioxidant N-acetyl-cysteine (NAC) potentiates ApoG2-induced apoptosis and cell killing. Our results show that ApoG2 induced protective autophagy in HCC cells, partly due to ROS generation, suggesting that antioxidant may serve as a potential chemosensitizer to enhance cancer cell death through blocking ApoG2-stimulated autophagy. Our novel insights may facilitate the rational design of clinical trials for Bcl-2-targeted cancer therapy.

Cell Death and Disease (2013) 4, e489; doi:10.1038/cddis.2013.17; published online 7 February 2013

Subject Category: Cancer

Human hepatocellular carcinoma (HCC) is the third most common malignancy and has a considerably high mortality rate. $^{1,2}$ Currently, treatment for patients with HCC mainly consists of traditional chemotherapy, which often yields unsatisfactory outcomes. ${ }^{3}$ Within this context, examination of novel molecular target compounds is a priority. Among these, inhibitors of the anti-apoptotic Bcl-2 family proteins exhibited broad antitumor activity both in vitro and in vivo. ${ }^{4}$

The anti-apoptotic Bcl-2 family proteins consist of $\mathrm{Bcl}-2$, $\mathrm{Bcl}-\mathrm{xL}, \mathrm{Mcl}-1, \mathrm{~A} 1$ and $\mathrm{Bcl}-\mathrm{W}$, which are generally integrated within the outer mitochondrial membrane. ${ }^{5}$ Overexpressed and dysregulated, anti-apoptotic Bcl-2 proteins are seen in various tumor types, such as prostate, non-Hodgkin's lymphoma, colorectal, breast, non-small cell lung cancer and also HCC. ${ }^{6}$ Abundant evidence has shown that suppression of anti-apoptotic $\mathrm{Bcl}-2$ proteins is a feasible strategy for cancer therapy. ${ }^{7}$

Apogossypolone (ApoG2) is a novel gossypol derivative designed by Ascenta Pharmaceuticals to reduce the toxicity of gossypol (a natural pan-inhibitor of anti-apoptotic Bcl-2 family proteins). ApoG2 has been reported as a potent inhibitor of $\mathrm{Bcl}-2, \mathrm{Bcl}-\mathrm{xL}$, and $\mathrm{Mcl}-1{ }^{8}$ ApoG2 blocks the interaction of $\mathrm{Bim}$ and $\mathrm{Bcl}-2$, and induces apoptosis in a number of human cancer cell lines. ${ }^{8-10}$ In addition, ApoG2 induces regression in several tumor xenograft models, and its maximum tolerated dose appeared to be less toxic than gossypol. ${ }^{10}$ Preclinical studies showed that ApoG2 had significant anti-lymphoma and anti-pancreatic cancer effects. $^{9,11}$

Autophagy has recently become an important issue in cancer research, and is emerging as a key regulator of death pathways. ${ }^{12}$ It has been reported that ApoG2 induced autophagy in several cancer cell lines such as breast, prostate and lymphoma, ${ }^{13-15}$ but the mechanisms have not been explored. In this study, we report that ApoG2 induces both Beclin-1- and reactive oxygen species (ROS)-mediated autophagy in HCC cells. Notably, we demonstrate that the suppression of autophagy using antioxidants enhances cell death induced by ApoG2, suggesting a new approach for combinational therapy in treating $\mathrm{HCC}$.

\section{Results}

ApoG2 induces autophagy in HCC cells. To test the ability of ApoG2 to induce autophagy in HCC cells, we analyzed LC-3 processing and localization, as well as

\footnotetext{
${ }^{1}$ Department of Biochemistry and Molecular Biology, College of Basic Medical Sciences, Third Military Medical University, Chongqing, China; ${ }^{2}$ Department of Educational College, Chongqing Normal University, Chongqing, China; ${ }^{3}$ Departments of Molecular Biosciences and Radiation Oncology, University of Kansas Cancer Center, University of Kansas, Lawrence, KS, USA and ${ }^{4}$ Key Laboratory of Biomedical Information Engineering of Education Ministry, School of Life Science and Technology, Xi'an Jiaotong University, Xi'an, China

${ }^{*}$ Corresponding author: F He and J Lian, Department of Biochemistry and Molecular Biology, College of Basic Medical Sciences, Third Military Medical University, 30 Gaotanyan, Shapingba, Chongqing 400038, People's Republic of China. Tel: + 8623 68752841; Fax: + 8623 68752262; E-mail: hefengtian06@yahoo.com.cn (FH) or E-mail: lianjiqin @sina.com (JL)

${ }^{5}$ These authors contributed equally to this work.

Keywords: ApoG2; ROS; autophagy; HCC

Abbreviations: ApoG2, apogossypolone; ROS, reactive oxygen species; NAC, N-acetyl-cysteine; HCC, hepatocellular carcinoma

Received 03.9.12; revised 07.1.13; accepted 08.1.13; Edited by T Bruner.
} 
autophagosome formation. Light chain 3 (LC3)-II is widely used as a marker of autophagy because its lipidation and specific recruitment to autophagosomes provide a shift from diffuse to punctate staining of the protein, and increases its electrophoretic mobility on gels compared with LC3-I. ${ }^{16}$ Significant conversion of LC3-I to LC3-II was detected in HepG2 and Hep3B cells treated for $24 \mathrm{~h}$ with $10 \mu \mathrm{m}$ ApoG2 (Figure 1a, rapamycin was used as positive control). Autophagy inhibitor chloroquine (CQ) blocks the fusion of autophagosomes and lysosomes, and results in the accumulation of LC3-II when combined with ApoG2 (Figure 1a). ApoG2-induced LC3-II conversion in HCC cells was doseand time-dependent (Figures $1 \mathrm{~b}$ and $\mathrm{c}$ ). Next, we examined the ApoG2-induced autophagy by LC3-GFP puncta formation. Recruitment of LC3-II to the autophagosomes is characterized by a punctate pattern of its subcellular localization. ${ }^{16}$ In HepG2 and Hep3B cells transfected with LC3-GFP, ApoG2-induced autophagy is evident by a punctate pattern of green-fluorescent LC3-GFP (Figure 1d, white arrows), whereas the DMSO control cells exhibited diffuse LC3-associated green fluorescence. Transmission electron microscopy (TEM) revealed an abundance of autophagosomes in HCC cells treated with ApoG2 (Figure 1e, black arrows). Collectively, these observations demonstrate that ApoG2 treatment activates autophagic flux in HCC cells.

ApoG2 interrupts the interaction of Beclin-1 and Bcl-2. To investigate the mechanism of autophagy induced by ApoG2, we used a co-immunoprecipitation (Co-IP) pull-down assay. Immunoprecipitation of Beclin-1 with specific antibodies pulled down $\mathrm{Bcl}-2$ from whole-cell lysates (WCL) (Figure 2a). This indicated that Bcl-2 and Beclin-1 were bound to each other in the cells, consistent with previous studies. ${ }^{17,18}$ Immunoprecipitation of Beclin-1 also pulled down Bcl-xL, but not $\mathrm{Mcl}-1$ (Figure 2a). ApoG2 treatment abolished this Bcl-2-Beclin-1 interaction as indicated by the disappearance of the specific pull-down bands (Figure 2a). ApoG2 also abrogated the binding of $\mathrm{Bcl}-\mathrm{xL}$ with Beclin-1 (Figure 2a). Furthermore, immunoprecipitation of Bcl-2 also pulled down Beclin-1, which can be interrupted by ApoG2 treatment (Figure 2b). Consistent with Rubinstein's study, Mcl-1 also binds with Atg12 directly (Supplementary Figure S1). However, ApoG2 treatment did not disrupt or increase the interaction of Atg12 and $\mathrm{Mcl}-1$ (Supplementary Figure S1). This data suggests a multifaceted BH3-mimetic capacity for ApoG2. Our data demonstrates that ApoG2 interrupts the interactions between Beclin-1 and $\mathrm{Bcl}-2 / \mathrm{Bcl}-\mathrm{xL}$, thus releasing the $\mathrm{BH}$-only pro-autophagic protein Beclin-1, which in turn triggers the autophagic cascade.

To further delineate the role of Beclin-1-Bcl-2 interaction in ApoG2-induced autophagy, we knocked down Beclin-1 protein levels by siRNA. Beclin-1 knockdown resulted in significant decrease but not complete disappearance of LC3-II conversion (Figure 2c) and positive punctuate GFP-LC3 HCC cell numbers (Figure $2 \mathrm{~d}$ ). These results demonstrate that the interaction of Bcl-2 and Beclin-1 has a key role in ApoG2triggered autophagy, but additional pro-autophagy pathways are also involved.

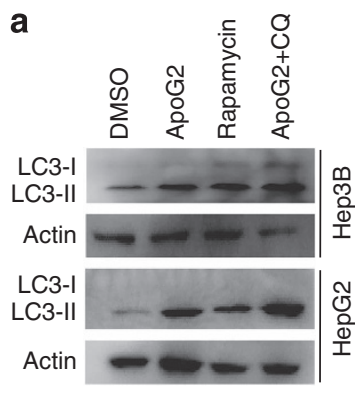

b

C
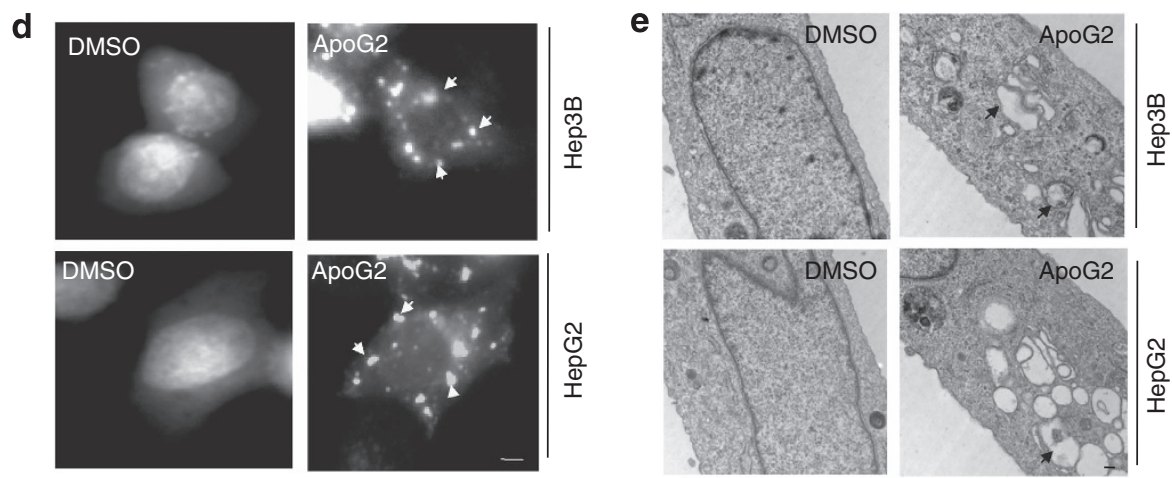

Figure 1 ApoG2 induces autophagy in HCC cells. (a) Cells were treated with $10 \mu \mathrm{m}$ ApoG2, $50 \mathrm{~nm}$ rapamycin or ApoG2 combined with $40 \mu \mathrm{m} \mathrm{CQ}$ for $24 \mathrm{~h}$. Then total protein was extracted and western blot was used to detect the expression of LC3. (b,c) Dose response for $24 \mathrm{~h}(\mathbf{b})$ and time course at $10 \mu \mathrm{m}$ concertration (c) of ApoG2induced autophagy in HCC cells. (d) Cells expressing LC3-GFP were treated with or without $10 \mu \mathrm{m}$ ApoG2 and processed for fluorescent microscopy $24 \mathrm{~h}$ later. White arrows indicate the characteristic punctate pattern of LC3-GFP, which occurs upon autophagic induction. (e) Representative electron micrograph images showing autophagic vacuoles (black arrows) following ApoG2 treatment 
a

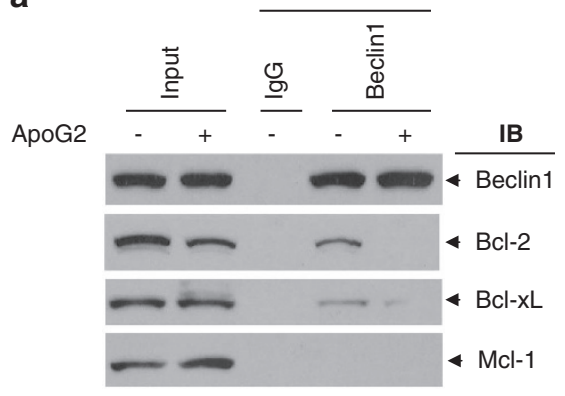

b

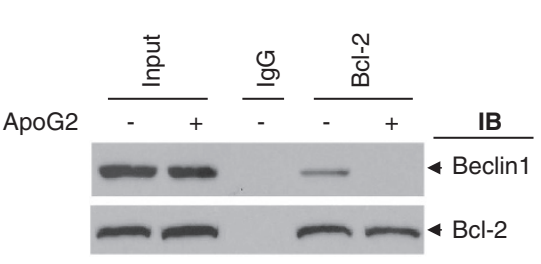

C

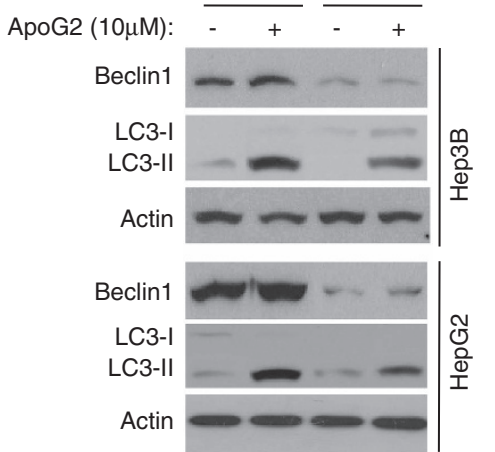

d

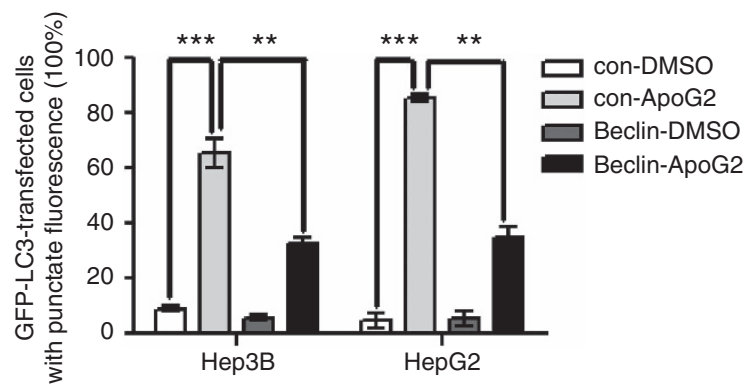

Figure 2 ApoG2 interrupts the interaction of Beclin-1 and Bcl-2. (a,b) Cell lysates were mixed with DMSO or $10 \mu \mathrm{m} \mathrm{ApoG2} \mathrm{for} 1 \mathrm{~h}$. Antibodies against Beclin-1 (a) or Bcl-2 (b) were added to immunoprecipitate the Beclin-1- or Bcl-2-containing complexes, and then immunoblotted for Bcl-2, Bcl-xL, Mcl-1 or Beclin-1. (c) Cells were transiently transfected with either control siRNA or siRNA-specific Beclin-1. Twenty-four hours after transfection, cells were treated with DMSO or ApoG2 for $24 \mathrm{~h}$, then subjected to immunoblot analysis for Beclin-1 and LC3. (d) Cells expressing LC3-GFP were transfected with either control siRNA or siRNA-specific Beclin-1. Twenty-four hours after transfection, cells were treated with DMSO or ApoG2 for $24 \mathrm{~h}$, a statistical analysis of the percentage of cells containing five or more GFP-LC3 puncta (50 green-fluorescent cells in one field, $n=5$ ). Data represent the mean value and from three independent experiments. ${ }^{\star \star} P<0.01,{ }^{* \star \star} P<0.001$

ApoG2 promotes ROS generation in HCC cells. As $\mathrm{BH} 3$ mimetics can activate multiple pro-autophagic pathways beside Beclin-1 activation ${ }^{19}$ and ApoG2 triggers the generation of cellular ROS in nasopharyngeal carcinoma, ${ }^{20}$ we next investigated whether ROS was triggered by ApoG2 in HCC cells by examining the intracellular levels of $\mathrm{GSH}, \mathrm{O}_{2}^{-}$and ROS. Consistent with the study on gossypol, ${ }^{21}$ treatment with ApoG2 significantly decreased intracellular GSH level (Figure 3a), and increased $\mathrm{O}_{2}^{-}$and ROS level (indicated by increased green fluorescence, Figures $3 b-d)$. Additionally, this phenotype was attenuated by antioxidant $\mathrm{N}$-acetylcysteine (NAC) co-treatment (Figures $3 a-d)$. These results indicate that ApoG2 promotes ROS generation in HCC cells.

\section{ApoG2 induces ROS-mediated autophagy in HCC cells.} To examine the role of ROS in ApoG2-induced autophagy, HCC cells were co-treated with ApoG2 and NAC. As shown in Figure $4 \mathrm{a}$, co-treatment with NAC decreased ApoG2induced LC3 conversion in HCC cells. This data indicates that ApoG2 induces ROS-mediated autophagy in HCC cells. Next, we determined whether additional pro-autophagy pathways are involved in ROS-mediated autophagy upon ApoG2 treatment. Consistent with the previous report, ${ }^{19}$ ApoG2 induced the hyperphosphorylation of ERK and JNK, as well as a reduction in the phosphorylation of mammalian target of rapamycin (mTOR) (Figure 4b). Co-treatment with NAC attenuated the effects of ApoG2 on ERK, JNK and
mTOR, suggesting that these pathways are regulated by ROS levels in HCC cells. As Bim and Atg5 are the targets of JNK, our data further demonstrated that ApoG2 treatment upregulated Bim and Atg5 in HCC cells (Supplementary Figure S2). The effect of ApoG2 on Bim and Atg5 can be suppressed by NAC co-treatment (Supplementary Figure S2). Recently, high mobility group box 1 (HMGB1) protein was discovered to be a critical pro-autophagic protein; furthermore, ROS promotes cytosolic translocation of HMGB1 and thereby enhances autophagic flux. ${ }^{22}$ Consistent with published data, treatment with ApoG2 increased the cytosolic HMGB1 level and decreased the nucleic HMGB1 level, which is then attenuated by NAC co-treatment in HCC cells (Figure 4c). Knockdown of endogenous HMGB1 also reduced ApoG2-induced autophagy in HCC cells (Figure 4d). We conclude that multiple pro-autophagic pathways are involved in ROS-mediated autophagy after ApoG2 treatment in HCC cells.

Antioxidant NAC enhances ApoG2-induced apoptotic cell death. To assess whether ROS-mediated autophagy is responsible for cell death, HCC cells were treated with ApoG2, NAC or their combination. As shown in Figures $5 \mathrm{a}$ and $b$, compared to ApoG2 alone, combination treatment with ApoG2 and NAC dramatically decreased cell viability and increased total cell death. The effect of ApoG2 and NAC on cell death was also found in human T-cell lymphoblast-like 

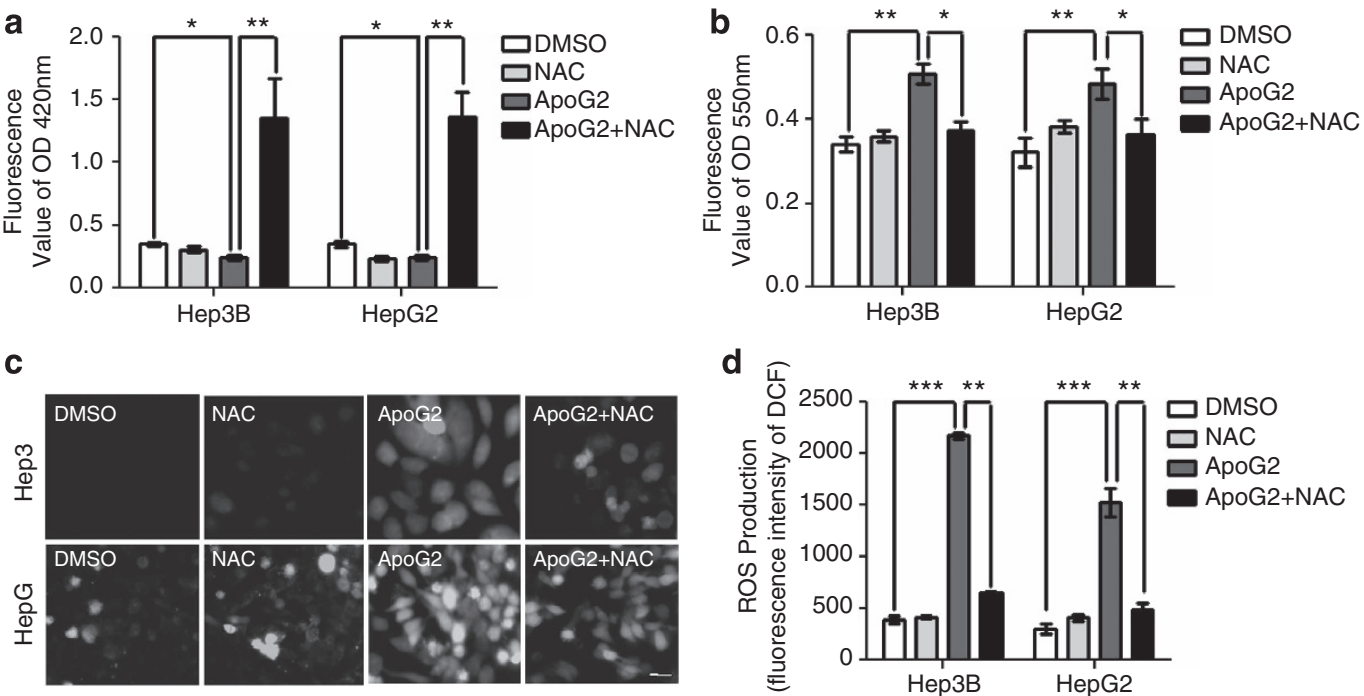

Figure 3 ApoG2 promotes ROS generation in HCC cells. Cells were pretreated with $10 \mathrm{~mm} \mathrm{NAC} \mathrm{or} \mathrm{PBS} \mathrm{for} 2 \mathrm{~h}$, and then incubated in $0.1 \%$ DMSO or $10 \mu \mathrm{M}$ ApoG2 for $24 \mathrm{~h}$. (a) Concentrations of GSH in the cells were measured by Elisa kit at $420 \mathrm{~nm}$. (b) Concentrations of $\mathrm{O}_{2}^{-}$in the cells were measured by Elisa kit at $550 \mathrm{~nm}$. (c) Representative fluorescent microscope images showing ROS level after treatment with DCF detection kit in HCC cells. (d) Fluorescence intensity of DCF in (c). Data represent the mean value and from three independent experiments. ${ }^{*} P<0.05,{ }^{* *} P<0.01,{ }^{* \star} P<0.001$
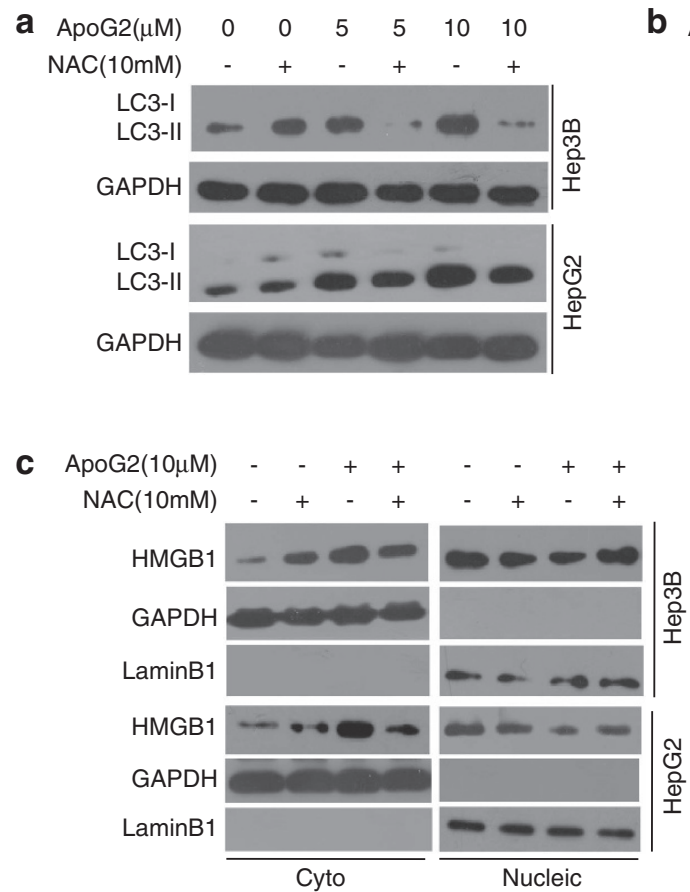

b ApoG2(10uM) - - + + - + + +

$\mathrm{NAC}(10 \mathrm{mM})-+-++\quad+\quad+$

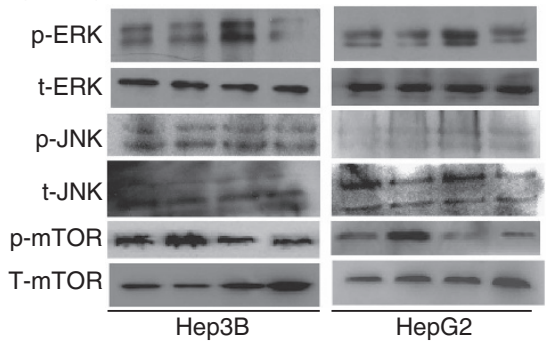

d

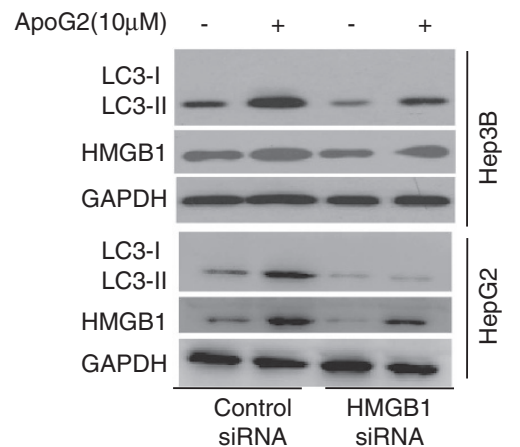

Figure 4 ApoG2 induces ROS-mediated autophagy in HCC cells. (a) Cells were treated with indicated concentrations of ApoG2 in the absence or presence of NAC for $24 \mathrm{~h}$. LC3 conversion was determined by western blot analysis. (b) Cells were treated with ApoG2 in the absence or presence of NAC for $24 \mathrm{~h}$. p-ERK, p-JNK, p-mTOR and t-ERK, t-JNK, t-mTOR were determined by western blot analysis. (c) Cells were treated as (b), then the fractions of nucleus and cytoplasm were separated, and were used to immunoblot analysis for HMGB1, LaminB1 or GAPDH. (d) Cells were transiently transfected with either control siRNA or siRNA-specific HMGB1. Twenty-four hours after transfection, cells were treated with DMSO or ApoG2 for $24 \mathrm{~h}$, then subjected to immunoblot analysis for HMGB1 and LC3

Jurkat cell, but not in human normal liver L02 cells and human embryonic kidney 293 cells (Supplementary Figures S3 and S4). These observations could be due to increased PARP cleavage (Figure 5c) and apoptotic cells (Figures $5 d$ and e). As Atg5 is a key regulator of autophagy and apoptosis, we further investigate the role of Atg5 in ApoG2induced cell death. Overexpression of Atg5 in HCC cells significantly reduced ApoG2-induced cell death and the 

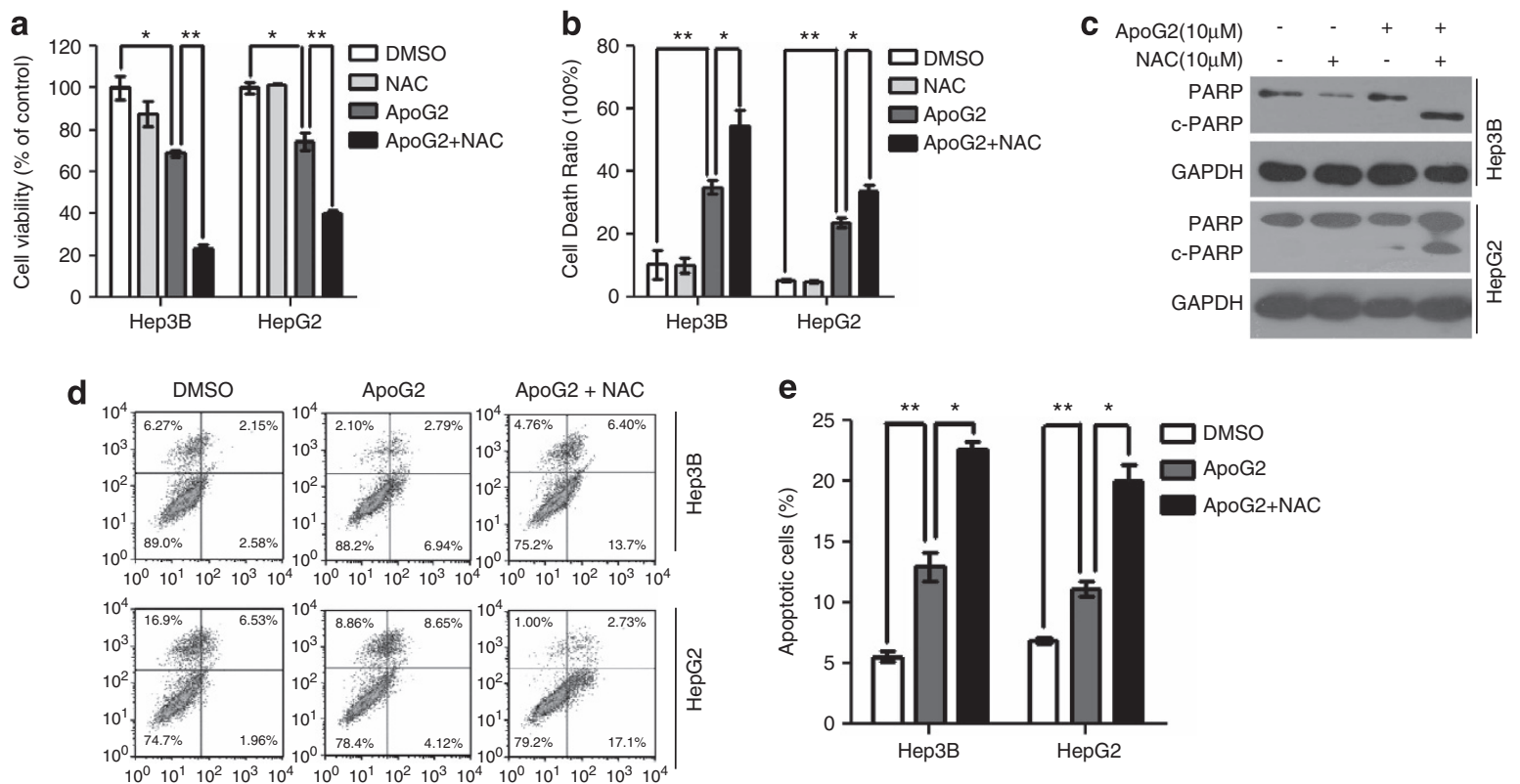

Figure 5 Antioxidant NAC enhances ApoG2-induced apoptotic cell death. (a) Cells were seeded at 2000 cells per well in 96 -well and were treated with $10 \mu \mathrm{m}$ ApoG2 in the absence or presence of $10 \mathrm{~mm} \mathrm{NAC} \mathrm{for} 24 \mathrm{~h}$. CCK8 was used to assess the cell viability. (b) Cells were treated as (a), trypan blue exclusion assay was used to check the total cell death. (c) Cells were treated as (a), PARP cleavage was determined by western blot analysis. (d,e) Cells were treated with ApoG2 or combined ApoG2 and NAC for $24 \mathrm{~h}$, apoptosis was measured by flow cytometry (d) and the percentage of apoptotic cells is calculated as the percentage of PI and Annexin-V double positive cells (e). Data represents the mean value and S.D. from three independent experiments. ${ }^{*} P<0.05,{ }^{* *} P<0.01$

cleavage of caspase-3 (Supplementary Figure S5), whereas downregulation of Atg5 in HCC cells dramatically enhanced cell death and caspase-3 cleavage (Supplementary Figure S5). NAC co-treatment downregulated the effect of Atg5 overexpression on ApoG2-induced cell death, but had no effect in Atg5 knockdown cells (Supplementary Figure S5). Our data strongly suggest that suppression of the ROSmediated autophagy induction potentiates apoptosis induction and cell killing by ApoG2.

\section{Discussion}

In the present study, we identified for the first time that antiapoptotic $\mathrm{Bcl}-2$ protein inhibitor, ApoG2, induced autophagy through Beclin-1- and ROS-dependent manners. Our working model (Figure 6) summarizes how ApoG2 induces autophagy by several means: abrogating the interaction of Beclin-1 and $\mathrm{BCl}-2 / \mathrm{xL}$, promoting ROS generation and therefore stimulating phosphorylation of ERK and JNK, increasing HMGB1 translocation and suppressing mTOR. Suppression of the ROS-mediated autophagy by antioxidant NAC potentiates apoptosis induction and cell killing by ApoG2.

The mechanisms of $\mathrm{BH}-3$ mimetics inducing autophagy through blocking the interaction of Beclin-1 and Bcl-2/xL are well documented. ${ }^{16,23}$ ApoG2, a new derivative of gossypol, in which the two aldehyde groups of gossypol are completely removed, interrupts the interaction of Beclin-1 and Bcl-2/xL but not Mcl-1. The same effect was found in compound gossypol. ${ }^{24}$ In spite of Mcl-1's ability to regulate autophagy through binding with Beclin- $1,{ }^{25}$ most of $\mathrm{BH}-3$ mimetics (except apogossypol derivative $\mathrm{BI}-97 \mathrm{C} 1)^{26}$ are unable to block it. The reasons may be due to the weak interaction

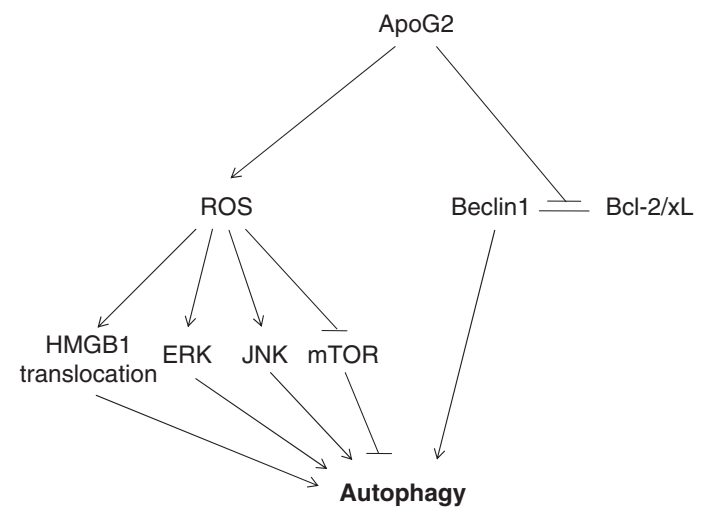

Figure 6 Working model of ApoG2-induced autophagy in HCC cells. ApoG2 induces autophagy by several means: abrogating the interaction of Beclin-1 and $\mathrm{BCl}-2 / \mathrm{xL}$, promoting ROS generation and therefore stimulating phosphorylation of ERK and JNK, increasing HMGB1 translocation and suppressing mTOR

between Beclin-1 and $\mathrm{Mcl}-1$, and the low affinity of $\mathrm{BH}-3$ mimetics with $\mathrm{Mcl}-1 .{ }^{27}$

Besides dissociation of Beclin-1 from its inhibitory factors, the study by Malik et al. ${ }^{19}$ showed that $\mathrm{BH}-3$ mimetics also activate multiple pro-autophagic signal transduction pathways. Our data showed that knockdown of Beclin-1 only partly suppressed ApoG2-induced autophagy, suggesting that there are additional pathways independent of Beclin-1 to induce autophagy. Consistently, ApoG2 causes the activations of ERK, JNK and HMGB1 translocation, and the inhibition of mTOR. Mammalian target of rapamycin (mTOR) is a conserved Ser/Thr kinase that inhibits autophagy by activation of the ATG1 and Akt kinases. ${ }^{28}$ MEK/ERK pathway 
regulates autophagy in Beclin-1-independent manners. ${ }^{29}$ Activation of JNK mediates the phosphorylation of $\mathrm{Bcl}-2$, Atg4 and p53, which contributes to autophagy induction. ${ }^{30,31}$ HMGB1 regulates autophagy partly through increasing transcriptional activities of JNK and ERK. ${ }^{32}$ Taken together, ApoG2 induces autophagy by Beclin-1-dependent and -independent manners.

ROS induces non-canonical autophagy by activating ERK and $\mathrm{JNK}^{33}$ inhibits mTOR signaling by activating AMPactivated protein kinase (AMPK) ${ }^{34}$ and promotes the translocation of oxidative HMGB1 from nucleus to cytoplasma. ${ }^{35}$ Previous data also reports that ApoG2 stimulates ROS production. $^{20}$ Therefore, we presume that ROS production may be responsible for the activation of multiple pro-autophagic signal pathways upon ApoG2 treatment. We conclude that ApoG2 stimulates ROS production by downregulating GSH level and upregulating $\mathrm{O}_{2}^{-}$in cells. Similar results were found in gossypol-treated cells, ${ }^{36}$ the mechanism may be through inhibiting electron transport in the mitochondria. ${ }^{37}$

The induction of ROS in HCC cells following ApoG2 treatment represents an alternative mechanism of ApoG2 cytotoxicity. The primary oxidation product of gossypol is gossypolone, and has been implicated in the formation of a redox system leading to additional free radical generation. ${ }^{38}$ Consistent with published literature, ${ }^{21}$ our study showed that ApoG2-induced ROS has a cytoprotective role, which may depend on ROS-mediated autophagy in HCC cells.

Autophagy, a process of intracellular organelle degradation, has either pro-survival or pro-death roles in cells when treated with a variety of chemotherapeutic drugs. ${ }^{39,40}$ Supported by our current study, targeting autophagy may become a new therapeutic strategy for overcoming autophagy-mediated chemoresistance. ${ }^{41} \mathrm{NAC}$ is well tolerated by humans and mice, ${ }^{42,43}$ and has been shown to block ROS generation in mouse tumors. ${ }^{44}$ Dietary supplements with high levels of NAC during ( - )-gossypol treatment may inhibit ROS generation in tumor cells, prevent oxidation of $(-)$-gossypol, and increase its efficacy and specificity against B-cell lymphoma. We conclude that the co-treatment of NAC with ApoG2 sensitizes HCC cells to cell death via an inhibition of cytoprotective authophagy. Furthermore, our study may provide a new direction on rational design of clinical trials as well as new strategies to overcome resistance to current cancer therapy.

Previous publication showed that ApoG2 induces apoptosis in HCC SMMC-7721 cells by downregulating anti-apoptotic proteins $\mathrm{Bcl}-2, \mathrm{Mcl}-1$ and $\mathrm{Bcl}-\mathrm{xL}$, upregulating pro-apoptotic protein Noxa, and promoting the activities of caspases- 9 and $-3 .{ }^{10}$ Cosistent with this report, ApoG2 induced apoptotic cell death in HepG2 and Hep3B cells. However, ApoG2 has little effect on human normal liver L02 cells and human embryonic kidney 293 cells. The reason may be due to the low expression level of anti-apoptotic protein $\mathrm{Bcl}-2 / \mathrm{xL}$ in these cells (Supplementary Figure S6). This point needs to be further validated.

\section{Materials and Methods}

Cell culture and reagents. Cell culture reagents and fetal bovine serum (FBS) were purchased from HyClone (Waltham, MA, USA). Human hepatocellular cell lines HepG2 and Hep3B were obtained from American Type Culture Collection and were cultured in DMEM nutrient mixture supplemented with 10\% FBS and antibiotics. ApoG2 was a gift kindly provided by the University of Michigan (Ann Arbor, MI, USA), and dissolved in DMSO at $20 \mathrm{~mm}$ as stock solution. NAC, CQ, rapamycin and acridine orange were from Sigma-Aldrich (Louis, MO, USA); Reactive Oxygen Species Assay Kit and Annexin V-FITC Apoptosis Detection Kit were purchased from Beyotime (Bejing, China). LC3 CDNA was kindly provided by Dr. N. Mizushima and T. Yoshimori at Osaka University, Japan.

Cell viability assay. CCK8 assay was used to assess cell viability. Cells were incubated in triplicate in 96 -well plates in the presence or absence of $(-)$ gossypol for $24 \mathrm{~h}$. Then the plates were centrifuged and the supernatant was discarded. Subsequently, each well was added with $90 \mu$ l RPMI 1640 medium containing $10 \%$ FBS and $10 \mu \mathrm{l}$ CCK8. The plate was incubated for $2 \mathrm{~h}$ and measured using microplate reader at $450 \mathrm{~nm}$.

Trypan blue exclusion assay. The treated cells were adequately suspended and $0.4 \%(\mathrm{w} / \mathrm{v})$ trypan blue solution was added at a volume ratio (the cell suspension to the trypan blue solution) of 9:1. Subsequently, the cells were counted under an optical microscope. Cells failing to exclude the dye were defined as dead cells. The total death rate $(\%)=$ numbers of dead cells/(numbers of living cells + numbers of dead cells) $\times 100$.

Western blot. Preparation of whole-cell protein lysates and western blot analysis were performed as described previously. ${ }^{45}$ Antibodies against microtubule-associated protein-1 LC3, PARP, HMGB1, JNK, ERK, mTOR, Lamin $B 1, B c l-x L$ were purchased from Cell Signaling (Boston, MA, USA) and the antibodies against Bcl-2, Mcl-1 and Beclin-1 were from Santa Cruz (Santa Cruz, CA, USA).

ROS generation detection. The intracellular ROS levels were measured by detecting the conversion of cell permeable 2, 7-dichlorofluorescein diacetate (DCFH-DA) to fluorescent dichlorofluorescein (DCF). Cells were seeded in 12-well plates and treated with the agents indicated for $12 \mathrm{~h}$. After washed with PBS for three times, cells were incubated with DCFH-DA at $37^{\circ} \mathrm{C}$ for $25 \mathrm{~min}$. Then the DCF fluorescence distribution was detected by fluorescence microplate reader at an excitation wavelength $(488 \mathrm{~nm})$ and emission wavelength $(525 \mathrm{~nm})$.

GFP-LC3 analysis. Cells were transfected with GFP-LC3 vector using Lipofectamine 2000 (Invitrogen Life Technologies, Carlsbad, CA, USA). Twenty-four hours later, cells were treated with drugs for $24 \mathrm{~h}$, then fixed in $4 \%$ formaldehyde for $10 \mathrm{~min}$. Cells were then washed three times with PBS and observed under a fluorescence microscope (OLYMPUS IX81, Tokyo, Japan) with $\times 40$ lens.

TEM. Preparation of the ultrathin sections were described previously. ${ }^{24}$ Ultrathin sections $(65 \mathrm{~nm})$ were examined using a TECNAI-10 TEM.

Co-immunoprecipitation experiments. HepG2 cells $\left(5 \times 10^{6}\right)$ were plated on $15 \mathrm{~cm}$ dishes and allowed to attach overnight. After treatment with either DMSO or $10 \mu \mathrm{M} A$ ApoG2 for $6 \mathrm{~h}$ at $37^{\circ} \mathrm{C}$, the whole-cell lysate was generated by collecting the supernatant at a low speed spin $(1000 \times g)$ to pellet nuclei after hypotonic lysis. WCL was precleared with protein A-agarose, then mixed with antibodies of Beclin-1 or Bcl-2 for $1 \mathrm{~h}$ at $4{ }^{\circ} \mathrm{C}$ (each IP reaction consisted of $1 \mathrm{mg}$ total protein). The immunoprecipitates were captured on protein $\mathrm{A}$-agarose and analyzed by immunoblotting with antibodies against Beclin-1, Bcl-2, Bcl-xL or Mcl-1, respectively.

Small interfering RNA transfection. siRNAs to Beclin-1, Hmgb1 or control siRNA were obtained from Dharmacon (Lafayette, CO, USA). Cells were plated in 6-well plates and transfected with siRNA (100 pmol per well) by Lipofectamine 2000 according to the manufacturer's manual. ApoG2 was added to the cells $36 \mathrm{~h}$ after transfection. For western blot, cells were collected after an additional $24 \mathrm{~h}$ culture.

Detection of apoptosis. Apoptosis was evaluated by Annexin V-FITC apoptosis detection kit following the manufacturer's instructions. Briefly, treated cells were harvested and incubated with AnnexinV-FITC and propidiumiodide (PI) at room temperature, respectively, for $10 \mathrm{~min}^{46}$ Subsequently, samples were analyzed by a FACScan flow cytometer. Hochest33258 staining was performed according to the manufacturer's instruction. 
Statistic analysis. The data were represented as the mean \pm S.D. from triplicate experiments. Two-way ANOVA was used to analyze the variance of different groups. A threshold of $P<0.05$ was defined as statistically significant.

\section{Conflict of Interest}

The authors declare no conflict of interest.

Acknowledgements. Grant support: This study was supported in part by Chongqing Natural Science Foundation (CSTC, 2011BB5030), and by the Scientific Funds of Third Military Medical University (2011XHG02).

1. El-Serag HB, Rudolph KL. Hepatocellular carcinoma: epidemiology and molecular carcinogenesis. Gastroenterology 2007; 132: 2557-2576.

2. Sherman M. Epidemiology of hepatocellular carcinoma. Oncology 2010; 78(Suppl 1): 7-10

3. Tanaka S, Arii S. Molecular targeted therapies in hepatocellular carcinoma. Semin Oncol 2012; 39: 486-492.

4. Zender L, Spector MS, Xue W, Flemming P, Cordon-Cardo C, Silke J et al. Identification and validation of oncogenes in liver cancer using an integrative oncogenomic approach. Cell 2006; 125: 1253-1267.

5. Levine B, Sinha S, Kroemer G. Bcl-2 family members: dual regulators of apoptosis and autophagy. Autophagy 2008; 4: 600-606.

6. Kang $\mathrm{MH}$, Reynolds $\mathrm{CP}$. Bcl-2 inhibitors: targeting mitochondrial apoptotic pathways in cancer therapy. Clin Cancer Res 2009; 15: 1126-1132.

7. Warr MR, Shore GC. Small-molecule Bcl-2 antagonists as targeted therapy in oncology. Curr Oncol 2008; 15: 256-261.

8. Sun Y, Wu J, Aboukameel A, Banerjee S, Arnold AA, Chen J et al. Apogossypolone, a nonpeptidic small molecule inhibitor targeting $\mathrm{Bcl}-2$ family proteins, effectively inhibits growth of diffuse large cell lymphoma cells in vitro and in vivo. Cancer Biol Ther 2008; 7 1418-1426.

9. Arnold AA, Aboukameel A, Chen J, Yang D, Wang S, Al-Katib A et al. Preclinical studies of Apogossypolone: a new nonpeptidic pan small-molecule inhibitor of $\mathrm{Bcl}-2, \mathrm{Bcl}-\mathrm{XL}$ and $\mathrm{Mc}-$ 1 proteins in follicular small cleaved cell lymphoma model. Mol Cancer 2008; 7: 20.

10. Mi JX, Wang GF, Wang HB, Sun XQ, Ni XY, Zhang XW et al. Synergistic antitumoral activity and induction of apoptosis by novel pan Bcl-2 proteins inhibitor apogossypolone with adriamycin in human hepatocellular carcinoma. Acta Pharmacol Sin 2008; 29: 1467-1477.

11. Banerjee S, Choi M, Aboukameel A, Wang Z, Mohammad M, Chen J et al. Preclinical studies of apogossypolone, a novel pan inhibitor of bcl-2 and $\mathrm{mcl}-1$, synergistically potentiates cytotoxic effect of gemcitabine in pancreatic cancer cells. Pancreas 2010; 39: 323-331.

12. Chen N, Karantza V. Autophagy as a therapeutic target in cancer. Cancer Biol Ther 2011 11: $157-168$

13. Niu X, Li S, Wei F, Huang J, Wu G, Xu L et al. Apogossypolone induces autophagy and apoptosis in breast cancer MCF-7 cells in vitro and in vivo. Breast Cancer 2012; doi:10.1007/s12282-012-0372-Z

14. Zhang XQ, Huang XF, Hu XB, Zhan YH, An QX, Yang SM et al. Apogossypolone, a novel inhibitor of antiapoptotic Bcl-2 family proteins, induces autophagy of PC-3 and $\mathrm{LNCaP}$ prostate cancer cells in vitro. Asian J Androl 2010; 12: 697-708.

15. Wei J, Kitada S, Stebbins JL, Placzek W, Zhai D, Wu B et al. Synthesis and biological evaluation of Apogossypolone derivatives as pan-active inhibitors of antiapoptotic B-cell lymphoma/leukemia-2 (Bcl-2) family proteins. J Med Chem 2010; 53: 8000-8011.

16. Maiuri MC, Criollo A, Tasdemir E, Vicencio JM, Tajeddine N, Hickman JA et al. BH3-only proteins and $\mathrm{BH} 3$ mimetics induce autophagy by competitively disrupting the interaction between Beclin 1 and Bcl-2/Bcl-X(L). Autophagy 2007; 3: 374-376.

17. Sinha S, Levine B. The autophagy effector Beclin 1: a novel BH3-only protein. Oncogene 2008; 27(Suppl 1): S137-S148.

18. Sinha S, Colbert CL, Becker N, Wei Y, Levine B. Molecular basis of the regulation of Beclin 1-dependent autophagy by the gamma-herpesvirus $68 \mathrm{Bcl} 2$ homolog M11. Autophagy 2008; 4: 8.

19. Malik SA, Orhon I, Morselli E, Criollo A, Shen S, Marino G et al. BH3 mimetics activate multiple pro-autophagic pathways. Oncogene 2011; 30: 3918-3929.

20. Zubair H, Khan HY, Ullah MF, Ahmad A, Wu D, Hadi SM. Apogossypolone, derivative of gossypol, mobilizes endogenous copper in human peripheral lymphocytes leading to oxidative DNA breakage. Eur J Pharm Sci 2012; 47: 280-286

21. Sikora MJ, Bauer JA, Verhaegen M, Belbin TJ, Prystowsky MB, Taylor JC et al. Antioxidant treatment enhances anti-tumor cytotoxicity of (-)-gossypol. Cancer Biol Ther 2008; 7: 767-776.

22. Tang D, Kang R, Livesey KM, Cheh CW, Farkas A, Loughran P et al. Endogenous HMGB1 regulates autophagy. J Cell Biol 2010; 190: 881-892.

23. Lian J, Karnak D, Xu L. The Bcl-2-Beclin 1 interaction in (-)-gossypol-induced autophagy versus apoptosis in prostate cancer cells. Autophagy 2010; 6: 1201-1203.

24. Lian J, Wu X, He F, Karnak D, Tang W, Meng Y et al. A natural BH3 mimetic induces autophagy in apoptosis-resistant prostate cancer via modulating Bcl-2-Beclin1 interaction at endoplasmic reticulum. Cell Death Differ 2011; 18: 60-71.

25. Germain M, Slack RS. MCL-1 regulates the balance between autophagy and apoptosis. Autophagy 2011; $7: 5$

26. Dash R, Azab B, Quinn BA, Shen X, Wang XY, Das SK et al. Apogossypol derivative BI97C1 (Sabutoclax) targeting Mcl-1 sensitizes prostate cancer cells to mda-7/LL-24mediated toxicity. Proc Natl Acad Sci USA 2011; 108: 8785-8790.

27. Voss V, Senft C, Lang V, Ronellenfitsch MW, Steinbach JP, Seifert V et al. The pan-Bcl-2 inhibitor (-)-gossypol triggers autophagic cell death in malignant glioma. Mol Cancer Res 2010; 8: 1002-1016.

28. Diaz-Troya S, Perez-Perez ME, Florencio FJ, Crespo JL. The role of TOR in autophagy regulation from yeast to plants and mammals. Autophagy 2008; 4: 851-865.

29. Wang J, Whiteman MW, Lian H, Wang G, Singh A, Huang D et al. A non-canonical MEK ERK signaling pathway regulates autophagy via regulating Beclin 1. J Biol Chem 2009; 284: 21412-21424.

30. Wei Y, Pattingre S, Sinha S, Bassik M, Levine B. JNK1-mediated phosphorylation of Bcl-2 regulates starvation-induced autophagy. Mol Cell 2008; 30: 678-688.

31. Li Y, Luo Q, Yuan L, Miao C, Mu X, Xiao W et al. JNK-dependent Atg4 upregulation mediates asperphenamate derivative BBP-induced autophagy in MCF-7 cells. Toxicol App Pharmacol 2012; 263: 21-31.

32. Zhao M, Yang M, Yang L, Yu Y, Xie M, Zhu S et al. HMGB1 regulates autophagy through increasing transcriptional activities of JNK and ERK in human myeloid leukemia cells. BMB Rep 2011; 44: 601-606.

33. Wong CH, Iskandar KB, Yadav SK, Hirpara JL, Loh T, Pervaiz S. Simultaneous induction of non-canonical autophagy and apoptosis in cancer cells by ROS-dependent ERK and JNK activation. PLoS One 2010; 5: e9996.

34. Yuan Y, Xue X, Guo R-B, Sun X-L, Hu G. Resveratrol enhances the antitumor effects of temozolomide in glioblastoma via ROS-dependent AMPK-TSC-mTOR signaling pathway. CNS Neurosci Ther 2012; 18: 536-546.

35. Kang R, Livesey KM, Zeh HJ 3rd, Lotze MT, Tang D. HMGB1 as an autophagy sensor in oxidative stress. Autophagy 2011; 7: 8 .

36. Sikora MJ, Bauer JA, Verhaegen M, Belbin TJ, Prystowsky MB, Taylor JC et al. Antioxidant treatment enhances anti-tumor cytotoxicity of (-)-gossypol. Cancer Biol Ther 2008; 7: 767-776.

37. Arinbasarova AY, Medentsev AG, Krupyanko VI. Gossypol inhibits electron transport and stimulates ROS generation in Yarrowia lipolytica mitochondria. Open Biochem J 2012; 6: $11-15$

38. Hu ZY, Wang J, Cheng G, Zhu XF, Huang P, Yang D et al. Apogossypolone targets mitochondria and light enhances its anticancer activity by stimulating generation of singlet oxygen and reactive oxygen species. Chin J Cancer 2011; 30: 41-53.

39. Dalby KN, Tekedereli I, Lopez-Berestein G, Ozpolat B. Targeting the prodeath and prosurvival functions of autophagy as novel therapeutic strategies in cancer. Autophagy 2010; 6: 322-329.

40. Baehrecke EH. Autophagy: dual roles in life and death? Nat Rev Mol Cell Biol 2005; 6 : 505-510.

41. Kondo Y, Kondo S. Autophagy and cancer therapy. Autophagy 2006; 2: 85-90.

42. Schrier BP, Lichtendonk WJ, Witjes JA. The effect of N-acetyl-L-cysteine on the viscosity of ileal neobladder mucus. World J Urol 2002; 20: 64-67.

43. Conesa EL, Valero F, Nadal JC, Fenoy FJ, Lopez B, Arregui B et al. N-acetyl-L-cysteine improves renal medullary hypoperfusion in acute renal failure. Am J Physiol Regul Integr Comp Physiol 2001; 281: R730-R737.

44. Shimamoto K, Hayashi H, Taniai E, Morita R, Imaoka M, Ishii Y et al. Antioxidant N-acetylL-cysteine (NAC) supplementation reduces reactive oxygen species (ROS)-mediated hepatocellular tumor promotion of indole-3-carbinol (I3C) in rats. J Toxicol Sci 2011; 36: 775-786.

45. Lian J, Ni Z, Dai X, Su C, Smith AR, Xu L et al. Sorafenib sensitizes (-)-gossypol-induced growth suppression in androgen-independent prostate cancer cells via Mcl-1 inhibition and Bak activation. Mol Cancer Ther 2011; 11: 416-426.

46. Yan HL, Xue G, Mei Q, Wang YZ, Ding FX, Liu MF et al. Repression of the miR-17-92 cluster by p53 has an important function in hypoxia-induced apoptosis. EMBO J 2009; 28: 2719-2732.

(c)

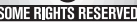
under the Creative Commons Attribution-NonCommercialShare Alike 3.0 Unported License. To view a copy of this license, visit http://creativecommons.org/licenses/by-nc-sa/3.0/ 